\title{
A comparative study of mid-infrared, UV-Visible and fluorescence spectroscopy in combination with chemometrics for the detection of adulteration of fresh olive oils with old olive oils
}

\author{
Oguz Uncu, Banu Ozen* \\ Food Engineering Department, Izmir Institute of Technology, Urla-Izmir, Turkey
}

\section{A R T I C L E I N F O}

\section{Keywords:}

Olive oil

Adulteration

FT-IR spectroscopy

UV-vis spectroscopy

Fluorescence spectroscopy

Chemometrics

\begin{abstract}
A B S T R A C T
The work aimed to detect and quantify adulteration of fresh olive oils with old olive oils from the previous harvest year by using different spectroscopic approaches in combination with chemometrics. Adulterated samples prepared in varying concentrations $(10-50 \%(\mathrm{v} / \mathrm{v}))$ were analyzed with fluorescence, Fourier transforminfrared (FT-IR), and ultraviolet-visible (UV-vis) spectroscopic methods. Orthogonal partial least square-discriminant analysis (OPLS-DA) and partial least squares (PLS) regression techniques were used for the differentiation of adulterated oils from the pure oils and prediction of adulteration levels, respectively. After the application of various pre-treatment methods, all of the OPLS-DA classification models generated for every spectroscopic technique successfully differentiated adulterated and non-adulterated oils with over $90 \%$ correct classification rate. FT-IR + UV-vis and fluorescence spectral data were also successfully used to predict adulteration levels with high coefficient of determinations for both calibration (0.94 and 0.98) and prediction (0.91 and 0.97 ) models and low error values for calibration (4.22\% and $2.68 \%$ ), and prediction (5.20\% and $2.82 \%$ ), compared to individual FT-IR and UV-vis spectroscopy were obtained. Therefore, FT-IR + UV-vis and fluorescence spectroscopy as being fast and environmentally friendly tools have great potential for both classification and quantification of adulteration practices involving old olive oil.
\end{abstract}

\section{Introduction}

Olive oil is a highly demanded product by the consumers in global scale due to its unique sensory characteristics, high nutritional value as well as its proven health benefits. These positive characteristics are mainly associated with the unique chemistry of olive oil which is mainly composed of monounsaturated fatty acids (mainly oleic acid) and minor components (phenolic compounds, $\alpha$-tocopherol and carotenoids) (Li \& Wang, 2018). Some of these constituents in olive oil are present in the highest level immediately after the extraction and there could be dramatic changes in their quantity during the storage mostly due to oxidative processes. As a result, "best before" date is critical for the quality of olive oil (Tena, Aparicio, \& García-González, 2018). An update in European Union regulation was done about olive oil labelling requirements in 2012 (EU, 2012). According to this regulation, harvest year can be placed on the label only if $100 \%$ of the product was obtained from the olives harvested in the same year. Therefore, mixing of the olive oils from the previous harvest with freshly extracted olive oils is regarded as an adulteration if the label indicates harvest year and a need arises to determine this type of adulteration to prevent unfair profit and to protect the consumers. However, detection methods which aim to differentiate old oils in fresh oils have not been thoroughly studied in the literature.

Detection of lower price seed and/or vegetable oils such as corn, sunflower and soybean oils (Jiménez-Carvelo, Osorio, Koidis, GonzálezCasado, \& Cuadros-Rodríguez, 2017; Sun, Lin, Li, Shen, \& Luo, 2015) and lower quality olive oils (refined or pomace olive oil) (Merás, Manzano, Rodríguez, \& de la Peña, 2018) in high quality olive oils have already been investigated by non-targeted fingerprinting techniques. In addition, classical targeted approaches which are based on wet chromatographic techniques of mainly gas chromatography (GC) (Jabeur, Drira, Rebai, \& Bouaziz, 2017; Jabeur, Zribi, \& Bouaziz, 2016) and high-performance liquid chromatography (HPLC) (Carranco, FarrésCebrián, Saurina, \& Núñez, 2018) and their combination (Jabeur et al., 2014) have been also used for the determination of these adulterants. Main disadvantages of these wet methods are their time consuming and waste producing nature. Rapid, simple, environmentally friendly and relatively cheap spectroscopic methods have been commonly used as

\footnotetext{
* Corresponding author.

E-mail address: banuozen@iyte.edu.tr (B. Ozen).
} 
alternatives to wet chemical methods in adulteration detection studies (Valli et al., 2016).

There is a growing interest of olive oil analysis by using fluorescence spectroscopy since it has comparably low detection limits with respect to other spectroscopic methods (Danezis, Tsagkaris, Camin, Brusic, \& Georgiou, 2016). Recently, this technique was used in the detection of different types of adulterants such as sunflower oil, (Ali et al., 2018), corn oil (Öztürk, Ankan, \& Özdemir, 2010), soybean oil (Tan et al., 2018), and lower quality olive oil (Dankowska \& Małecka, 2009; Merás et al., 2018) in extra virgin olive oil.

Fourier-transform infrared (FT-IR) spectroscopy was also used successfully along with chemometric methods in many different adulteration studies such as differentiation of olive oils from soybean oil, sunflower oil, rapeseed (canola) oil, corn oil, peanut oil, sesame oils, camellia oil, walnut oil and grapeseed oils (Jiménez-Carvelo et al., 2017; Sun et al., 2015; Rohman, Che Man, Ismail, \& Hashim, 2017).

Ultraviolet-visible (UV-vis) spectroscopy comes forward as a quite easy to use technique. However, there are relatively few studies in the literature about the detection of adulteration with UV-vis spectroscopy. This technique was used to quantify lower grade olive oil in extra virgin olive oil (Torrecilla, Rojo, Domínguez, \& Rodríguez, 2010) as well as different blends of olive oil with corn, soybean, and sunflower oils (Jiang, Zheng, \& Lu, 2015).

Although there are several successful examples of olive oil adulteration detection studies using different spectroscopic methods, differentiation of mixtures of olive oils such as mixtures from different olive varieties, mixtures of refined and extra virgin olive oils or mixtures of fresh and old olive oils is generally a more challenging task. Therefore, it is important to test the capabilities of these techniques for these cases. To the best of our knowledge there is a few preliminary studies in the literature about the detection of adulteration concerning mixing of old olive oils with fresh olive oils. FT-IR was used to detect limited number of adulterated samples in one study (Hirri et al., 2015) and laser diode-based fluorescence spectroscopy was also used in another research (Torreblanca-Zanca et al., 2019). In addition, a recent study employed different classification methods in analyzing fluorescence spectra to determine freshness of olive oils as expired or nonexpired (Dankowska \& Kowalewski, 2019). However, there is not any study which compares the performances of different spectroscopic approaches about this emerging issue.

Hypothesis of this research is that fresh olive oil could be differentiated from old olive oil in a mixture by using fluorescence, FT-IR and UV-vis and the combination of FT-IR and UV-vis spectroscopies; moreover, quantification of different levels of adulterant is also possible with these spectroscopic methods when they are used along with multivariate statistical approaches. Therefore, it was aimed to investigate the effectiveness of different spectroscopic techniques individually and also in combination to detect this type of fraud in a fast way with minimal chemical waste.

\section{Materials and methods}

\subsection{Olive oil samples}

Fresh olive oil samples obtained in 2016 harvest year were analyzed immediately after the production whereas olive oils from 2015 harvest year were used as old olive oil samples after one year of storage. Olive oils were from the different regions (14 different locations for fresh olive oils and 5 different locations for old olive oils) of Turkey. Twenty different fresh and 5 different old oils were used in the analyses and 4 fresh and 5 old olive oils were mixed with each other in cross combinations and the rest of the fresh samples (16 samples) were independently used. As a result, 100 adulterated samples in five different concentrations from $10 \%$ to $50 \%$ level with $10 \%$ increments (20 samples for each level) were prepared with a total volume of $10 \mathrm{~mL}$ by mixing samples with a vortex.

\subsection{Determination of free fatty acid content, $K$ values and fatty acid profile}

Basic quality parameters, free fatty acid (FFA) and specific extinction coefficients (K232 and K270) as well as fatty acid profile of the olive oil samples were determined according to European Official Methods of Analysis (EEC, 1991).

FFA value was determined by titrating dissolved $20 \mathrm{~g}$ of olive oil sample in $150 \mathrm{~mL}$ diethyl ether-ethanol solution (1:1) with standardized $0.1 \mathrm{~mol} \mathrm{~L}^{-1}$ solution of potassium hydroxide until a change in indicator color (phenolphthalein). Results were expressed in terms of $\%$ oleic acid.

Absorbance values of $0.25 \mathrm{~g}$ of the olive oil samples diluted to $25 \mathrm{~mL}$ with cyclohexane were measured at 232 and $270 \mathrm{~nm}$ with a spectrophotometer (Shimadzu UV-2450 Spectrophotometer, Japan) using the pure cyclohexane as the blank.

Fatty acid profile of the methyl esterified olive oil samples was determined by a GC with flame-ionization detector (FID) (Agilent 6890, Agilent Technologies, USA) possessing an auto-sampler (Agilent 7863) with a split/splitless inlet. As a capillary column, HP-88 with dimensions of $100 \mathrm{~m} \times 0.25 \mathrm{~mm} \mathrm{ID} \times 0.2 \mathrm{~mm}$ (Agilent, USA) was used. Experimental conditions were as follows; $1 \mathrm{~mL}$ eluent were injected with a split ratio $1 / 50$, helium was used as a carrier gas at constant $2 \mathrm{~mL} \mathrm{~m}^{-1}$ flow, injection and detector temperature were set to $250^{\circ} \mathrm{C}$ and $280^{\circ} \mathrm{C}$, respectively. Temperature program of oven was kept at $120^{\circ} \mathrm{C}$ for $10 \mathrm{~min}$ and then increased to $220^{\circ} \mathrm{C}$ with a rate of $3^{\circ} \mathrm{C} \mathrm{m}-1$ and maintained at the same temperature for $5 \mathrm{~min}$. The sample chromatogram peaks were compared with the retention times of fatty acid methyl ester (FAME) mix standards. The results including major individual fatty acids, total saturated fatty acids (SFA), total monounsaturated fatty acids (MUFA), and total polyunsaturated fatty acids (PUFA) were given as the relative percentage of FAME. Three replicates of each measurement were recorded.

\subsection{Spectroscopic methods}

\subsubsection{FT-IR spectroscopy}

Mid-infrared spectra between 4000 and $650 \mathrm{~cm}^{-1}$ of the olive oil samples were recorded by using PerkinElmer Spectrum 100 FT-IR spectrometer (PerkinElmer Inc., USA) equipped with a deuterated triglycine sulphate detector (DTGS). As a sampling technique horizontal attenuated total reflectance (HATR) accessory with ZnSe crystal was used. Scan speed, resolution, and number of scans for each spectrum were adjusted as $1 \mathrm{~cm} \mathrm{~s}^{-1}, 4 \mathrm{~cm}^{-1}$, and 64 respectively. The spectra for each sample was taken twice. After each analysis, the sampling crystal was cleaned with hexane, ethanol and deionized water.

\subsubsection{UV-vis spectroscopy}

UV-visible spectrophotometer (Shimadzu UV-2450 Spectrophotometer, Japan) was used to obtain the spectra of olive oil samples between 200 and $800 \mathrm{~nm}$. Absorbance was measured with fast scan speed in a macro type polystyrene cuvette $(12.5 \times 12.5 \times 45 \mathrm{~mm})$ having $10 \mathrm{~mm}$ light path by using air as the blank. Sampling interval and slit width were set to $2.0 \mathrm{~nm}$ and $5.0 \mathrm{~nm}$, respectively. Duplicated spectra were obtained for each olive oil sample.

\subsubsection{Fluorescence spectroscopy}

Fluorescence spectra of the olive oil samples were acquired with the LS-55 fluorescence spectrometer (PerkinElmer Inc., USA) equipped with a pulsed xenon lamp. The slit width was adjusted to $5 \mathrm{~nm}$ for both excitation and emission. Data interval for scan and integration time was set to $0.5 \mathrm{~nm}$ and $0.2 \mathrm{~s}$, respectively. These parameters were selected to obtain the best resolution with optimal signal-to-noise ratio.

For each excitation wavelength $(320,330,340$ and $350 \mathrm{~nm})$ fluorescence emission spectra were recorded twice for each sample between 300 and $800 \mathrm{~nm}$ simultaneously by using a quartz cell. An excitation wavelength at $350 \mathrm{~nm}$ was selected both in the construction of 
classification and prediction models.

\subsection{Multivariate statistical analysis}

In order to handle the large data clusters obtained from the spectroscopic measurements, multivariate statistical tools were utilized in both classification and prediction. SIMCA 14.0 software (Umetrics, Sweden) was used for the data analyses. The whole spectra from FT-IR $\left(4000-650 \mathrm{~cm}^{-1}\right)$, UV-vis $(200-800 \mathrm{~nm})$, and fluorescence (300$800 \mathrm{~nm}$ ) spectroscopy measurements were used in the analyses. In addition, low level data fusion was applied to FT-IR and UV-vis spectroscopic data to obtain a single matrix and this combined form was also used in both classification and prediction models. Low level data fusion is a basic combination method relied on concatenating data sets obtained from different instruments into a large single matrix and could be used in generating classification or prediction models. Rows and colons of the matrix correspond to samples and signals (variables), respectively (Borràs et al., 2015).

Prior to the model development, replicated spectroscopic data were averaged and then appropriate pre-processing techniques were used to remove the undesirable instrumental and experimental variations (Engel et al., 2013). Pre-processing techniques could be divided into two main categories as signal enhancement and signal correction methods (Moros, Garrigues, \& de la Guardia, 2010). Mean-centering and unit variance scaling were applied as a signal enhancement strategy in the construction of all models. Advanced signal correction algorithms such as first derivative (FD), second derivative (SD), Savitzky-Golay (SG), wavelet denoising techniques (WDTs), multiplicative scatter correction (MSC), and orthogonal signal correction (OSC) were used individually and in appropriate combinations (S-G:MSC, FD:S-G:MSC, and WDTs:OSC) for the development of the specific models. FD and SD of the spectroscopic data were calculated from moving quadratic submodels with 15 data point long and the distance between each data point is set to 1 excluding the edge effects. As a wavelet function Daubechies-10 was chosen, and confidence interval was selected as $99.5 \%$. Selection of the suitable pre-processing technique was accomplished with the trial and error method. For this purpose, different preprocessing techniques were applied and the best performing one was selected with respect to their classification and prediction efficiencies in terms of the statistical parameters provided in Tables 1 and 2, respectively (Engel et al., 2013).

For the classification and quantification, pre-treated data set of each spectroscopic technique was randomly divided into calibration and validation sets comprising $2 / 3$ and $1 / 3$ number of the data set,

\section{Table 1}

OPLS-DA models of different spectroscopic methods in classification of adulterated and fresh samples (the number of samples are shown in parenthesis).

\begin{tabular}{|c|c|c|c|c|c|c|}
\hline Method & Pre-treatment ${ }^{\mathrm{a}}$ & LVs & $\mathrm{R}_{\text {cal. }}^{2}$ & $\mathrm{R}_{\mathrm{cv}}^{2}$ & $\begin{array}{l}\% \mathrm{CC}_{\mathrm{cal}}{ }^{\mathrm{b}} \\
(\mathrm{n}=80)\end{array}$ & $\begin{array}{l}\text { \%CC }{ }_{\text {pred. }}{ }^{c} \\
(\mathrm{n}=40)\end{array}$ \\
\hline & FD & $1+3$ & 0.98 & 0.53 & 100 & 93 \\
\hline \multirow[t]{3}{*}{ FT-IR } & WDTs:FD & $1+3$ & 0.97 & 0.58 & 100 & 85 \\
\hline & SD & $1+2$ & 0.97 & 0.42 & 100 & 90 \\
\hline & FD & $1+3$ & 0.98 & 0.98 & 100 & 83 \\
\hline \multirow[t]{3}{*}{ UV-vis } & WDTs:FD & $1+3$ & 0.98 & 0.97 & 100 & 83 \\
\hline & SD & $1+4$ & 0.99 & 0.98 & 100 & 100 \\
\hline & FD & $1+4$ & 0.99 & 0.66 & 100 & 98 \\
\hline \multirow[t]{3}{*}{ FT-IR + UV-vis } & WDTs:FD & $1+3$ & 0.98 & 0.65 & 100 & 85 \\
\hline & SD & $1+2$ & 0.97 & 0.58 & 100 & 88 \\
\hline & FD & $1+8$ & 0.95 & 0.70 & 100 & 90 \\
\hline \multirow[t]{2}{*}{ Fluorescence } & WDTs:FD & $1+8$ & 0.98 & 0.71 & 100 & 95 \\
\hline & SD & $1+7$ & 0.90 & 0.68 & 100 & 89 \\
\hline
\end{tabular}

\footnotetext{
${ }^{\text {a }}$ FD: first derivative, SD: second derivative, WDTs:FD combination of wavelet denoising techniques and first derivative.

b Average correct classification rate for calibration.

c Average correct classification rate for prediction (external validation).
}

respectively. The calibration data set was used to generate the corresponding model. An optimal model with respect to the latent variables (LVs) was chosen by internal validation (cross validation) which was applied as leave-one-out cross validation (LOO-CV) to avoid over and/ or under fitting of the model (Riedl, Esslinger, \& Fauhl-Hassek, 2015). The optimal number of LVs obtained from 7-fold cross validation revealed the model complexity and the percentage of correct classification for the optimized number of LVs provides the classification accuracy (Engel et al., 2013).

In classification studies, orthogonal partial least square-discriminant analysis (OPLS-DA) was used to visualize the separation of adulterated and fresh olive oil samples by using pre-treated data. In OPLS-DA analysis, a dummy Y matrix (variable vector) consisting of class 1 and class 2 (adulterated and non-adulterated (fresh) samples, respectively) was correlated with X matrix (spectral data) (Sen \& Tokatli, 2016). The results of the OPLS-DA analysis are given in the form of a misclassification table. Both cross and external validation techniques were used to determine correct classification and misclassification (known as rejection or error) rate (Riedl et al., 2015). The correct classification rate (\%CC) was determined when an examined oil sample from a defined olive oil class (as adulterated or non-adulterated) have a prediction value between 0.5 and 1.5; otherwise, it was considered as a misclassification (Hirri, Bassbasi, Platikanov, Tauler, \& Oussama, 2016). In addition, other performance parameters such as number of latent variables (LVs), coefficient of determination for calibration $\left(\mathrm{R}_{\text {cal }}^{2}\right)$ and goodness of prediction $\mathrm{Q}^{2}\left(\mathrm{R}_{\mathrm{cv}}^{2}\right)$ were determined for each classification model constructed with different spectroscopic data. These values were evaluated by automatic fitting function available in the SIMCA software.

Prediction for the quantification of the varying levels of adulteration $(0-50 \% \mathrm{v} / \mathrm{v})$ were conducted with partial least squares (PLS) regression. Basically, PLS regression was used to correlate spectroscopic absorbance of each adulterated and non-adulterated sample (X block) with the percentages of adulterant and non-adulterant olive oil ( $Y$ block) (Gurdeniz \& Ozen, 2009). The prediction ability of the generated PLS models were investigated with several performance parameters such as $R_{\text {cal }}^{2}$ for calibration, $R_{\text {cv }}^{2}$ for cross validation, $R_{\text {pred }}^{2}$ for external validation. Error values as root mean square error of prediction (RMSEP), root mean square error of calibration (RMSEC), root mean square value of cross-validation (RMSECV) were also used in the performance evaluation. $\mathrm{R}^{2}$ values should be close to 1 while error values should be small and close to each other in order to minimize error as low as possible by sustaining balance between generated error values in terms of magnitude and to obtain a robust prediction model (Uncu \& Ozen, 2015). Additional parameters such as residual predictive deviation (RPD) for external validation and slope of the calibration models were also used to evaluate the model. The RPD value stands for the ratio of standard deviation of predicted values to RMSEP values revealing the predictive ability of the corresponding model (Riedl et al., 2015). The RPD values were calculated according to formula provided in the literature (Ozturk, Yucesoy, \& Ozen, 2012). In RPD evaluation, values lower than 2.0 are considered to be insufficient for prediction while values between 2.0 and 2.5 are used for approximate quantitative predictions. Values between 2.5 and 3.0 and values higher than 3.0, on the other hand, indicate good and excellent predictions, respectively (Tamaki \& Mazza, 2011).

\section{Results and discussion}

\subsection{Chemical characteristics of olive oil}

Free fatty acid and specific extinction (K232 and K270) values of the olive oil samples were determined to evaluate the general quality of the samples. Average acidity (\%), K232, and K270 values of fresh olive oil samples used in mixing studies were $0.40 \pm 0.12,2.18 \pm 0.21$, and $0.20 \pm 0.01$, respectively while the same parameters for the old olive 
Table 2

Statistical parameters of PLS regression models for prediction of adulteration by different spectroscopic methods.

\begin{tabular}{|c|c|c|c|c|c|c|c|c|c|c|}
\hline Method & Pre-treatment ${ }^{\mathrm{a}}$ & LVs & $\mathrm{R}^{2}$ cal. & $\mathrm{R}^{2} \mathrm{cv}$. & $\mathrm{R}^{2}$ pred. & RMSEC & RMSECV & RMSEP & RPD & Slope \\
\hline \multirow[t]{4}{*}{ FTIR } & FD & 3 & 0.90 & 0.58 & 0.54 & 5.44 & 12.02 & 11.53 & 1.5 & 0.90 \\
\hline & S-G:MSC & 4 & 0.67 & 0.45 & 0.46 & 10.14 & 12.62 & 13.34 & 1.3 & 0.67 \\
\hline & FD:S-G:MSC & 4 & 0.97 & 0.48 & 0.57 & 2.97 & 13.95 & 11.64 & 1.5 & 0.97 \\
\hline & WDTs:OSC & 9 & 0.96 & 0.77 & 0.84 & 3.45 & 10.19 & 7.01 & 2.5 & 0.96 \\
\hline \multirow[t]{4}{*}{ UV-vis } & FD & 6 & 0.73 & 0.61 & 0.66 & 9.35 & 10.56 & 10.06 & 1.7 & 0.73 \\
\hline & S-G:MSC & 5 & 0.69 & 0.59 & 0.60 & 9.93 & 10.89 & 11.03 & 1.5 & 0.69 \\
\hline & FD:S-G:MSC & 4 & 0.72 & 0.62 & 0.57 & 9.30 & 10.36 & 11.44 & 1.6 & 0.72 \\
\hline & WDTs:OSC & 6 & 0.86 & 0.80 & 0.80 & 6.78 & 8.02 & 7.76 & 2.2 & 0.86 \\
\hline \multirow[t]{4}{*}{ FTIR + UV-vis } & FD & 3 & 0.92 & 0.63 & 0.62 & 5.10 & 11.33 & 10.56 & 1.6 & 0.92 \\
\hline & S-G:MSC & 5 & 0.77 & 0.49 & 0.64 & 8.57 & 12.45 & 10.29 & 1.7 & 0.77 \\
\hline & FD:S-G:MSC & 6 & 0.99 & 0.70 & 0.61 & 1.49 & 10.91 & 10.70 & 1.6 & 0.99 \\
\hline & WDTs:OSC & 5 & 0.94 & 0.85 & 0.91 & 4.22 & 6.96 & 5.20 & 3.2 & 0.94 \\
\hline \multirow[t]{4}{*}{ Fluorescence } & FD & 4 & 0.73 & 0.32 & 0.37 & 9.15 & 16.61 & 13.61 & 1.3 & 0.73 \\
\hline & S-G:MSC & 10 & 0.79 & 0.44 & 0.49 & 8.31 & 13.07 & 12.28 & 1.4 & 0.79 \\
\hline & FD:S-G:MSC & 5 & 0.72 & 0.40 & 0.29 & 9.43 & 13.82 & 15.05 & 1.1 & 0.72 \\
\hline & WDTs:OSC & 9 & 0.98 & 0.95 & 0.97 & 2.68 & 6.52 & 2.82 & 6.2 & 0.98 \\
\hline
\end{tabular}

a FD first derivative, S-G:MSC combination of Savitzky-Golay and multiplicative scatter correction, FD:S-G:MSC combination of first derivative with SavitzkyGolay and multiplicative scatter correction, WDTs:OSC combination of wavelet denoising techniques and orthogonal signal correction.

oil samples were $0.92 \pm 0.29,2.30 \pm 0.32$, and $0.19 \pm 0.10$, orderly.

Average major fatty acids values (\%) of fresh olive oil samples were determined as follows; palmitic acid $13.79 \pm 0.97$, stearic acid $3.04 \pm 0.34$, oleic acid $69.63 \pm 1.83$, linoleic acid $10.74 \pm 1.46$, linolenic acid $0.76 \pm 0.11$, SFA $17.56 \pm 1.46$, MUFA $70.94 \pm 2.10$, and PUFA $11.50 \pm 1.57$. While the same parameters for old olive oil samples were determined as $14.09 \pm 2.05 \%$ palmitic acid, $2.68 \pm 0.13 \%$ stearic acid, $68.94 \pm 3.16 \%$ oleic acid, $11.56 \pm 3.24 \%$ linoleic acid, $0.74 \pm 0.07 \%$ linolenic acid, $17.41 \pm 2.30 \%$ SFA, $70.29 \pm 3.39 \%$ MUFA, and $12.30 \pm 3.31 \%$ PUFA.

All the studied samples were in the limits of quality standards of European Union regulation on olive oil (EU, 2016). Fresh olive oil samples were graded as extra virgin olive oils while old olive oil samples were at lower grades.

\subsection{Spectral evaluation}

Typical spectra of all the studied olive oil samples obtained with different spectroscopic techniques are shown in Fig. 1. The FT-IR spectra of the samples (Fig. 1a) are dominated by the peaks at distinct wavelengths of 2924, 2852, 1743, 1463, 1377, 1238, 1163, 1114, 1099 and $721 \mathrm{~cm}^{-1}$ (Sinelli, Cosio, Gigliotti, \& Casiraghi, 2007). Absorbances at 2924 and $2852 \mathrm{~cm}^{-1}$ wavelengths are due to $-\mathrm{CH}_{2}$ asymmetric and symmetric stretching vibrations, respectively. The major peaks at $1743 \mathrm{~cm}^{-1}$ followed by 1463 and $1377 \mathrm{~cm}^{-1}$ are associated with $\mathrm{C}=\mathrm{O}$ stretching, $\mathrm{CH}_{2}$ and $\mathrm{CH}_{3}$ scissoring vibrations, respectively. The rest of the peaks at $1238,1163,1114,1099 \mathrm{~cm}^{-1}$ are relevant with C-O stretching vibration while a small peak at $721 \mathrm{~cm}^{-1}$ are correlated with $\mathrm{CH}_{2}$ rocking mode (Sinelli et al., 2007).

UV-vis spectra of the olive oil samples are shown in Fig. 1b. Absorption spectra of the olive oil samples have specific peaks around 230-270 $\mathrm{nm}$ indicating the presence of conjugated dienes and trienes of unsaturated fatty acids. Moreover, $300-400 \mathrm{~nm}$ band was correlated with a variety of polyphenols (Mignani, Ciaccheri, Mencaglia, \& Cimato, 2012). A shift in the positions of the peaks and/or the absence of the peaks in the current study compared to above assignments could be related to differences in the quality, varietal and geographical differences of olive oil samples with respect to investigated samples in the literature as well as measurement parameters. In addition, carotenoids as one of the color pigments are responsible for the absorption between 430 and $460 \mathrm{~nm}$ and peak at $670 \mathrm{~nm}$ is attributed to chlorophylls and their derivatives (Mignani et al., 2012).

Fluorescence emission spectra of the olive oil samples are shown in
Fig. 1c and these spectra reveal three regions of interest around $350 \mathrm{~nm}$ due to specific excitation together with $400-600 \mathrm{~nm}$, and $650-750 \mathrm{~nm}$. Bands between 600 and $700 \mathrm{~nm}$ in emission possessed well known relationship with chlorophylls $a$ and $b$ and pheophytins a and b. Bands at $250-400 \mathrm{~nm}$ are correlated with $\alpha$-tocopherol and phenolic compounds while $400-600 \mathrm{~nm}$ emission spectral range could be attributed to vitamin $B_{2}$ and carotenoids as well as oxidation products of fatty acids, especially conjugated hydroperoxides, are found in the range of 440-470 nm (Ali et al., 2018; Dupuy et al., 2005).

Spectra obtained from each of these spectroscopic methods were further investigated to observe for any visual differences between a fresh sample and adulterated ones. The differences in FT-IR spectra were not easy to recognize visually. On the other hand, visual inspection revealed noticeable differences between the spectra of adulterated and fresh olive oil samples obtained with UV-vis and fluorescence spectroscopy.

Main differences in UV-spectra are observed in the peaks attributed to carotenoids $(400-500 \mathrm{~nm})$ and chlorophylls $(670 \mathrm{~nm})$ (Fig. 1b). Both chlorophylls and carotenoids are pigments which are affected from environmental conditions such as light and temperature and are converted into other forms and/or degraded during storage. Therefore, differences in UV-spectra of old oil containing samples could be associated with the changes in the pigment composition of the samples. The changes in pigment composition could be attributed to the oxidation of these compounds during storage (Ali et al., 2018).

Fluorescence emission spectra of the olive oil samples at varying adulteration levels are provided in Fig. 1c. Fluorescence intensity at distinct wavelengths $(400-500 \mathrm{~nm})$ increased with increasing adulteration level and this could be correlated with the formation of oxidation products of fatty acids such as hydroperoxides emitted around 450 nm (Lleó, Hernández-Sánchez, Ammari, \& Roger, 2016). However, fresh olive oil samples have higher intensity at $650-750 \mathrm{~nm}$ compared to adulterated ones and this difference could be attributed to change in chlorophyll content having negative linear relationship with oxidation products (Hernández-Sánchez, Lleó, Ammari, Cuadrado, \& Roger, 2017).

\subsection{Discrimination of fresh olive oils from adulterated oils}

OPLS-DA models were created with the data from each spectroscopic technique and with the combination of FT-IR and UV-vis spectral data and Table 1 shows the results of statistical parameters for the models obtained with the application of various spectral pre-treatments. Best models were obtained with FD of FTIR, FTIR + UV-vis and fluorescence spectroscopy while SD of UV-vis spectral data resulted in 


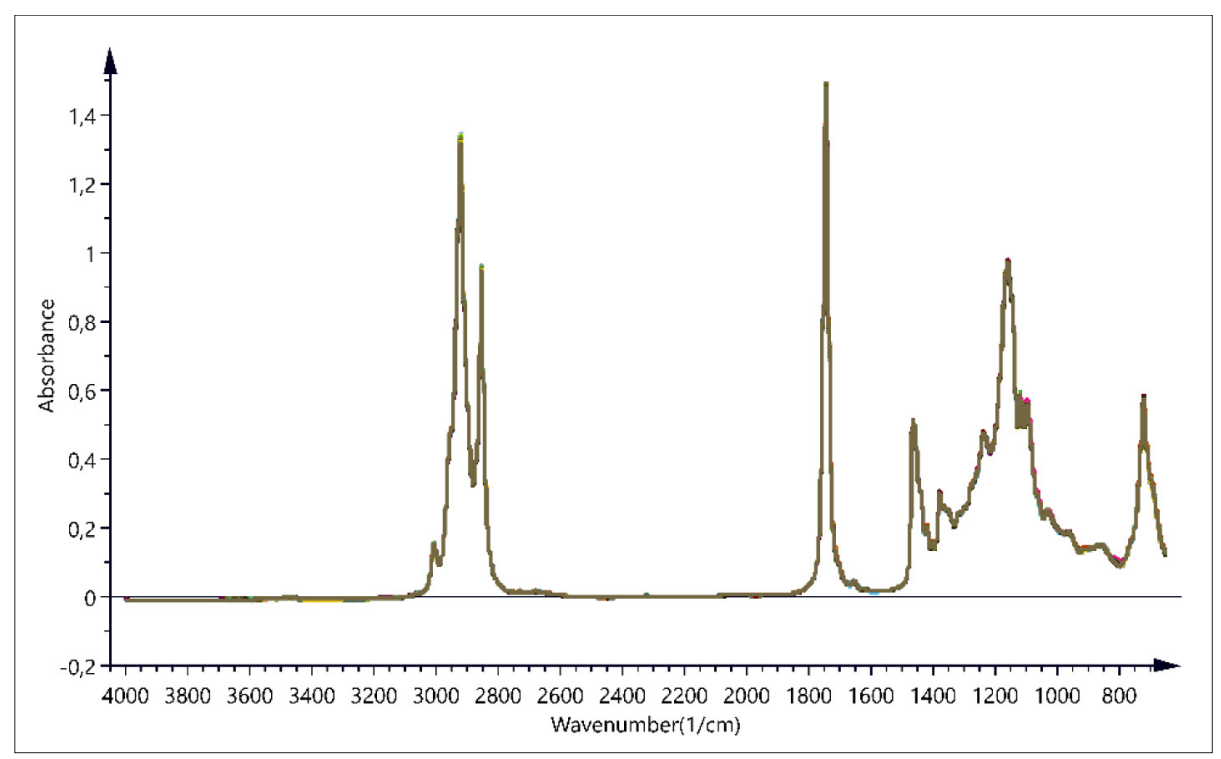

(a)

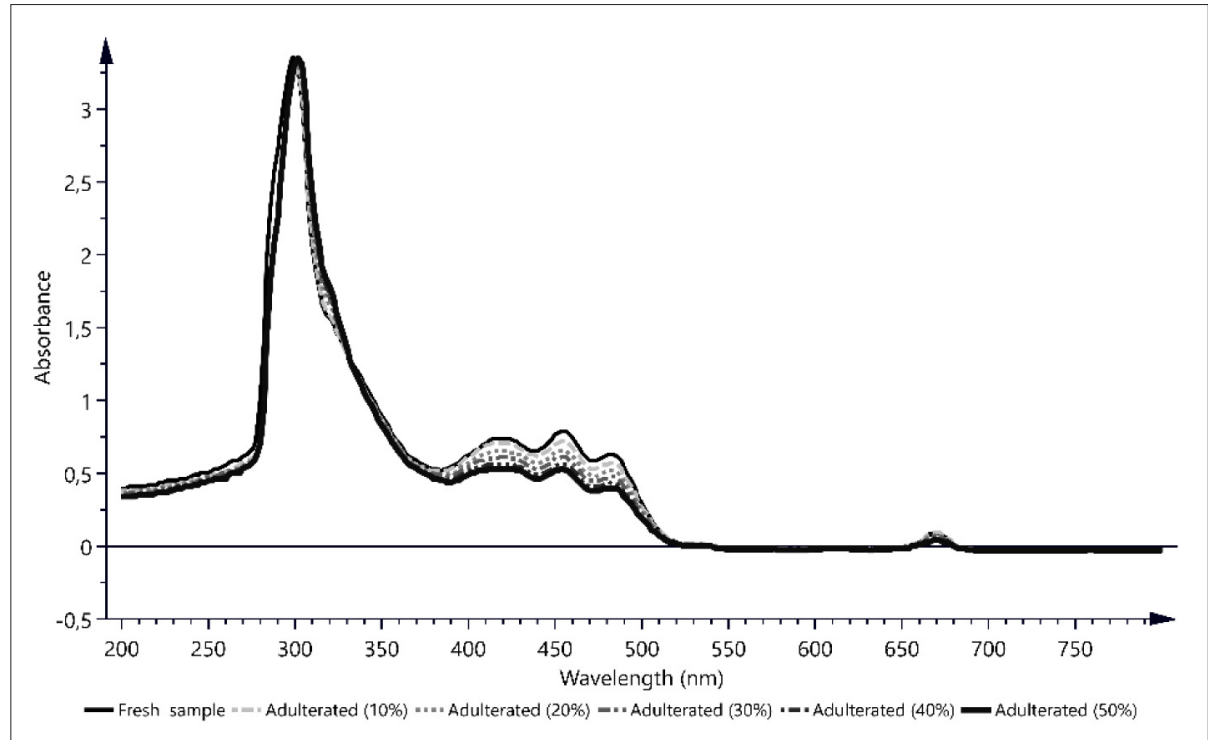

(b)

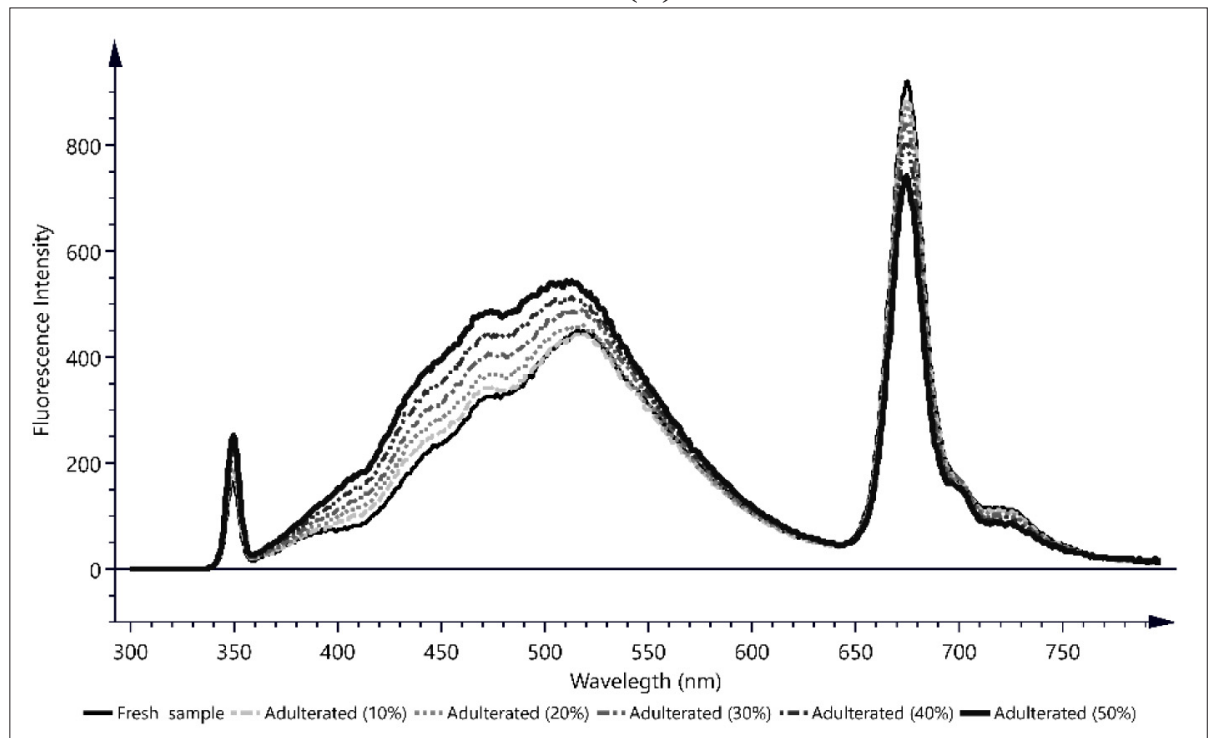

(c) 
Fig. 1. (a) FT-IR, (b) UV-vis and (c) fluorescence spectra of the olive oil samples.

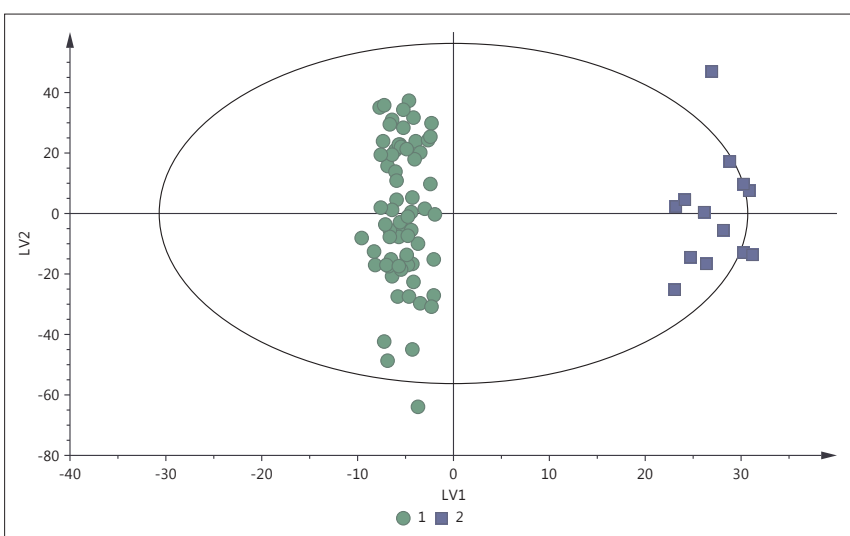

(a)

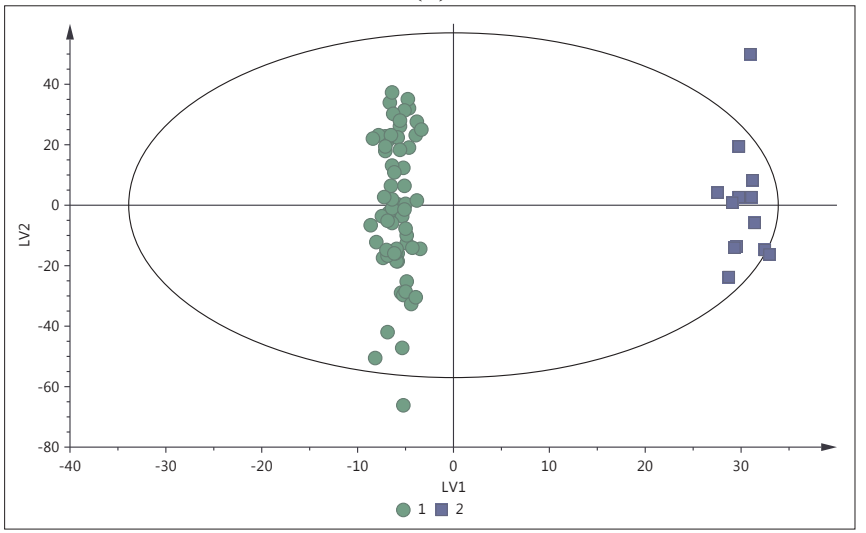

(c)

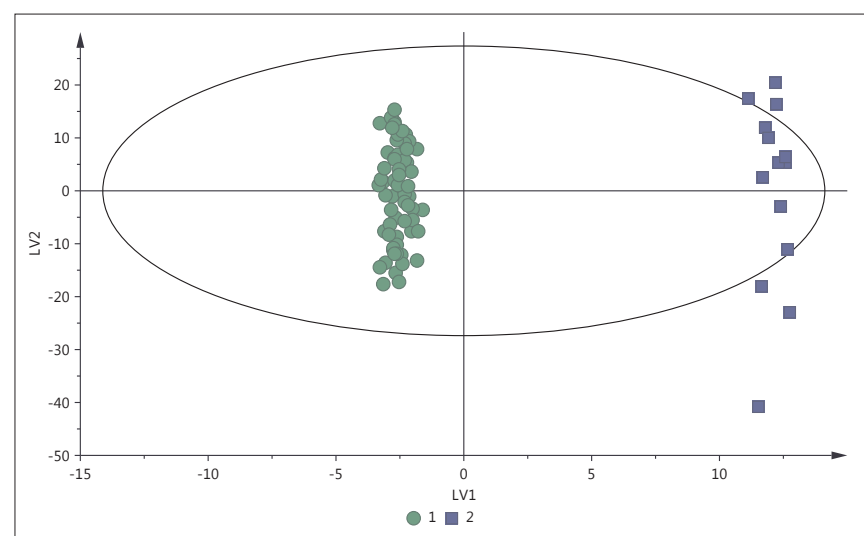

(b)

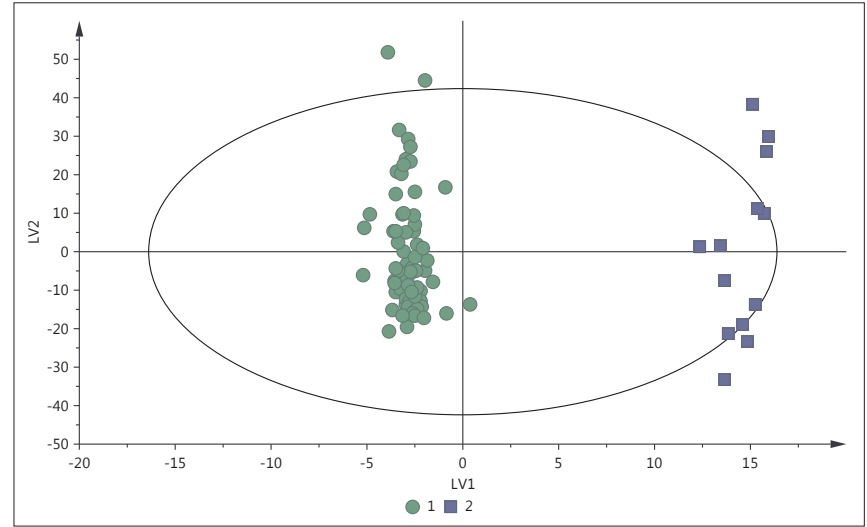

(d)

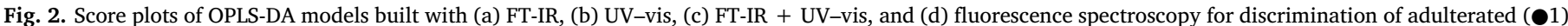
and fresh olive oil samples ( $\square 2)$.

the most successful differentiation. Each model was comprising a calibration and external validation set and the number of the samples in calibration and validation is 80 and 40 out of total 120 samples (100 adulterated and 20 fresh samples), respectively (Table 1). Although it might look like there is an unbalance between the numbers of adulterated (100) and non-adulterated (20) samples there is still enough number of non-adulterated samples to form a class in OPLS-DA model. Classification could be also performed by using each adulteration percentages as a different class. However, it was thought that assigning all adulteration levels to a single class is a more realistic approach. This is because of that it is generally impossible to know the adulteration concentrations of external samples that are brought to the control laboratories and constructed model allows detection of mixing regardless of adulteration percentages. OPLS-DA score plots of each calibration model are provided in Fig. 2 which shows the scattering of two classes as adulterated and fresh samples (non-adulterated).

As it could be seen from Table 1, OPLS-DA model of FD of FT-IR spectra provided the best differentiation of fresh olive oil samples from adulterated ones with the average correct classification rate of $100 \%$ and $93 \%$ (out of 40 sample; 1 sample misclassified as adulterated and 2 samples misclassified as fresh samples) in calibration and validation sets, respectively. The OPLS-DA model was built with 1 predictive and 3 orthogonal components. Other statistical parameters such as high $\mathrm{R}^{2}$ values for calibration and cross validation sets further confirm the classification ability of the model (Table 1). According to score plot (Fig. 2a), fresh samples located on the right side of the score plot are separated from adulterated samples with respect to the first latent variable (LV1) explaining $49 \%$ of the total variation. Furthermore, variable importance for the projection (VIP) values are also evaluated to determine the most significant wavelengths in differentiation of adulteration. VIP parameter is increasingly preferred in the model evaluation since it provides the most compact model interpretation compared to loading weights and regression coefficients (GalindoPrieto, Eriksson, \& Trygg, 2015). VIP values greater or close to 1 are considered as influential in the explanation of classification and prediction models (Uncu \& Ozen, 2015). The highest VIP values are obtained at around $1723 \mathrm{~cm}^{-1}$ which could be associated with stretching of $\mathrm{C}=\mathrm{O}$ (free fatty acids) groups (Hirri et al., 2016) as well as fingerprint region $\left(1464-983 \mathrm{~cm}^{-1}\right.$ ) and around $723 \mathrm{~cm}^{-1}$ (Jolayemi, Tokatli, Buratti, \& Alamprese, 2017). In the literature, there is only one study in which limited number of old olive oil samples (lampante) were separated from fresh (extra virgin) samples by using discriminant analysis (PLS-DA) of FT-IR data (Hirri et al., 2015).

Score plot of OPLS-DA model constructed with SD of UV-vis absorbance spectra is shown in Fig. 2b. A clear separation was obtained between fresh and adulterated samples in the calibration set (100\%) as well as in the external validation set with correct prediction rate of $100 \%$ (Table 1). LV1 was effective in classification by separating each class of olive oil samples to the left and the right of the score plot (Fig. 2b). The highest VIP values for the constructed model are found as around $260-290,470$ and $680 \mathrm{~nm}$ and these values correspond to the presence of conjugated dienes and trienes (oxidation products), carotenoids and chlorophyll derivatives, respectively. To the best of our knowledge, there is no comparable literature about the differentiation of old and fresh olive oil samples by using UV-vis spectroscopy. Until so far, studies with UV-vis spectroscopy have been based on the 
quantification of the adulteration of extra virgin olive oil with lower grade olive oils (Torrecilla et al., 2010) as well as binary and ternary mixtures of monovarietal extra virgin olive oils (Aroca-Santos, Cancilla, Pérez-Pérez, Moral, \& Torrecilla, 2016).

Combination of two spectroscopic methods as FT-IR + UV-vis is also investigated for any improvement that could be attributable to the data fusion in the classification of the samples. Prior to combining the data, first derivative of both spectra were taken individually and then they were fused. The fused data set provided the best OPLS-DA model and score plot of this model is shown in Fig. 2c. According to statistical results listed in Table 1, combined data have higher classification power than the model of FT-IR spectroscopic data and also have comparable success with UV-vis data. The model was built with 1 predictive and 4 orthogonal components explaining $56 \%$ of the overall model according to LV1. The measure of fit for calibration and cross validation are $99 \%$ and $66 \%$, respectively. The OPLS-DA model correctly separated all samples from two classes in the calibration set (100\%) and also correctly predicted all samples for each class in the external validation set except one misclassified sample from the adulterated set (98\%) (Table 1).

Fluorescence spectroscopy was also used in the differentiation of adulterated samples. De-noised fluorescence spectra were further pretreated with FD transformation prior to model construction. The OPLSDA score plots (Fig. 2d) revealed a good separation between adulterated and fresh olive oil samples which are scattered in the negative and positive sides of the LV1, respectively. The correct classification rates for both calibration and validation sets are satisfactorily high as $100 \%$ and $95 \%$ (2 samples misclassified as fresh samples), respectively. Certain wavenumbers around $435-500 \mathrm{~nm}$ and $670 \mathrm{~nm}$ could be correlated with higher VIP values in comparison to the rest of the wavelengths. These bands could be attributed to conjugated hydroperoxides and chlorophyll content, respectively (Ali et al., 2018); therefore, these compounds are most likely responsible for the differentiation of fresh olive oil from adulterated ones. As far as we know, there was only two very recent study in the literature using laser diode induced excitation to differentiate fresh and old olive oil samples successfully (Torreblanca-Zanca et al., 2019; Lastra-Mejías et al., 2019). Most of the fluorescence studies have been focused on the detection of lower grade olive oil (Merás et al., 2018) and authenticity confirmation and geographical origin determination (Jiménez-Carvelo, Lozano, \& Olivieri, 2019).

To sum up, all of the studied models are found to be quite successful in differentiation of adulteration with old olive oil samples. All the calibration models built with different spectroscopic techniques are $100 \%$ successful in adulteration detection while external validation models are also promising with decreasing order of correct classification rate for UV-vis, FT-IR + UV-vis, fluorescence, and FT-IR as 100\%, $98 \%, 95 \%$, and $93 \%$, respectively. Presence of oxidation products and change in pigment content caused differentiation of fresh olive oils adulterated with old olive oil from fresh olive oils.

\subsection{Prediction studies}

Quantification of adulterant level (0-50\% v/v) in fresh olive oil samples was conducted by applying PLS algorithm to the calibration and external validation data sets from each spectroscopic technique. Statistical results of each spectroscopic method as well as the combination of FT-IR and UV-vis are provided in Table 2. Different preprocessing techniques and appropriate combinations were used in model development and it was found out that OSC:WDTs provided better results compared with the rest of the transformations (Table 2). OSC was also reported as a more successful pre-processing technique compared to other methods in the literature (Cen \& He, 2007). Therefore, models developed by the OSC in combination with WDTs will be explained in more detail. Prediction performance of the models were evaluated by some critical internal and external as well as cross validation parameters such as regression coefficients $\left(R^{2}\right)$ and error values (RMSE) (Table 2). A model must have high $\mathrm{R}^{2}$ values and low RMSE values to have high predictive ability (Gurdeniz \& Ozen, 2009).

First approach was using FT-IR data set to quantify adulteration level. The model was constructed using $9 \mathrm{LVs}$ with relatively high $\mathrm{R}^{2}$ values for calibration (0.96), cross validation (0.77) and prediction, (0.84) and comparably low error values of $3.45 \%$ for calibration, $10.19 \%$ for cross validation, and $7.01 \%$ for prediction as well as robust RPD value of 2.5 were also obtained for this model (Table 2). There is only one preliminary study in the literature predicting limited number of lower quality olive oil (lampante) in fresh olive oil by FT-IR spectroscopy successfully with $\mathrm{R}^{2}$ of 0.999 and error values lower than $1 \%$ (Hirri et al., 2015). Results of the present study have lower performance due to higher prediction error compared to the previous study. In the former study, smaller number of samples $(n=45)$ were used and the old olive oil samples were in a more degraded condition as lampante virgin oil with free fatty acidity of $3.28 \%$ compared to the samples having an average $0.92 \%$ of free fatty acid value in the present study.

PLS model of UV-vis spectra have moderate prediction power including $6 \mathrm{LVs}$ along with acceptable $\mathrm{R}^{2} \geq 0.80$ and close error values with approximate RPD value of 2.2 (levels of RPD are defined section 2.4) (Table 2). UV-vis spectroscopy had similar prediction power with FT-IR spectroscopy. In the literature, there is not any study which used UV-vis spectral data in prediction of this type of adulteration. UV-vis studies were performed for determining the level of adulteration of extra virgin olive oil with refined olive oil and refined olive-pomace oil (Torrecilla et al., 2010) and also for the quantification of binary and ternary mixtures of monovarietal extra virgin olive oils (Aroca-Santos et al., 2016).

FT-IR + UV-vis data are quite successful in the prediction of varying levels of old olive oil samples in fresh ones with robust statistical parameters $\left(\mathrm{R}_{\text {cal }}^{2}=0.94, \quad \mathrm{R}_{\text {pred }}^{2}=0.91, \quad \mathrm{RMSE}-\mathrm{C}=4.22 \%\right.$, RMSEP $=5.20 \%$, and RPD $=3.2$ ) (Table 2$)$. For better visualization of the prediction model, PLS regression plot is presented in Fig. 3a. It is clear that the data fusion approach is more successful in the quantification of adulteration compared to individual methods (FT-IR or UV-vis) (Table 2). In a recent study, it was also reached to a similar conclusion about the prominent improvement in the model prediction power for the quantification of rapeseed oil in olive oil blends by near infrared (NIR) and mid infrared (MIR) spectroscopy (Li, Xiong, \& Min, 2019).

PLS regression plot of fluorescence spectroscopic data for the prediction model built with 9 LVs was presented in Fig. $3 \mathrm{~b}$. High $\mathrm{R}^{2}$ values for both calibration (0.98) and external validation (0.97) sets as well as lower error values for the same data sets $(2.68 \%$ and $2.82 \%$, respectively) showed that fluorescence spectroscopy is a promising tool in the detection of old olive oils mixed with fresh olive oils (Table 2). Results of the present study is in accordance with two very recent study, both of which used laser diode induced excitation. These studies were able to detect expired extra virgin olive oil with error values around $1.5 \%$ and lower than $10 \%$ by using different statistical approaches of intelligent non-linear model based on a supervised artificial neural network (Torreblanca-Zanca et al., 2019) and a linear model relying on chaotic parameters (Lastra-Mejías et al., 2019), respectively.

In summary, fluorescence and combination of FT-IR and UV-vis spectroscopic data provided better results in the quantification of adulteration than two other individual spectroscopic data. Therefore, it is recommended to use combined data rather than individual UV-vis and FT-IR methods alone to determine this type of adulteration. In addition, fluorescence spectroscopic data also resulted in robust prediction models with similar statistical parameters as fused data. Detection errors for both techniques were lower than $10 \%$. Moreover, fluorescence spectroscopy performed slightly better than combined spectroscopy in terms of determination limit as well as other statistical parameters. It was found that $10 \%$ detection limit is satisfactory for this type of adulteration since fraudsters could make little profit lower than 


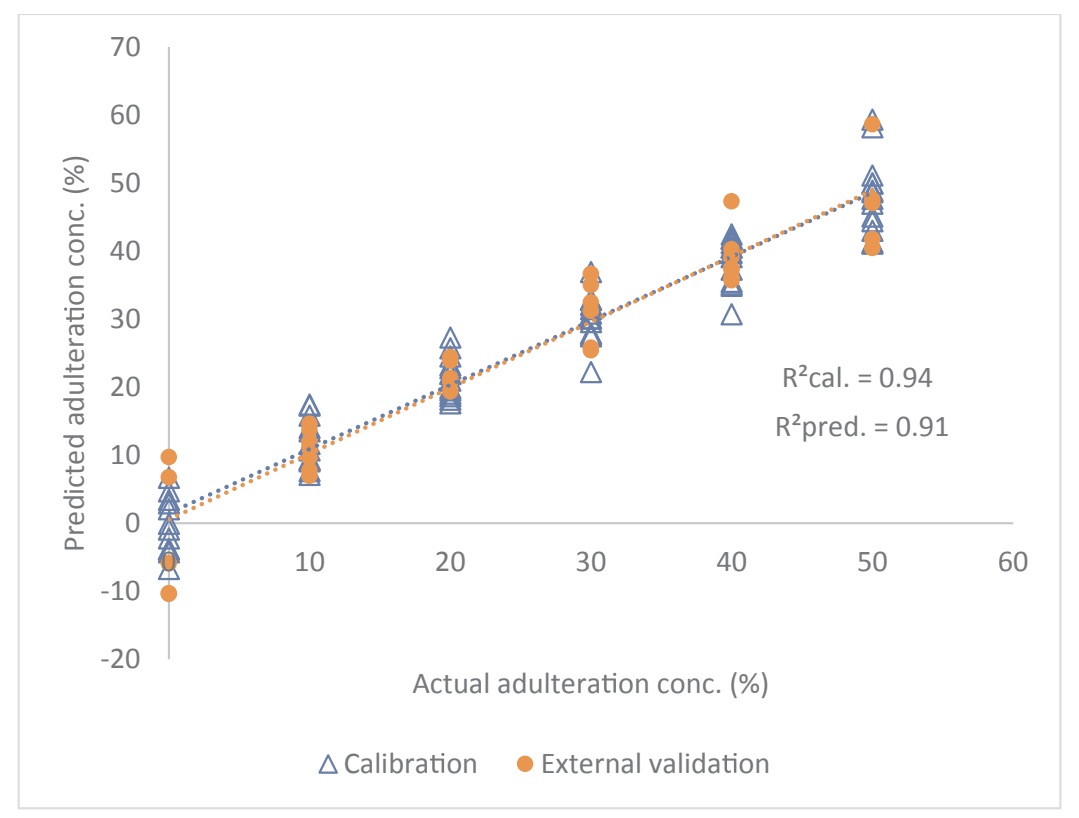

(a)

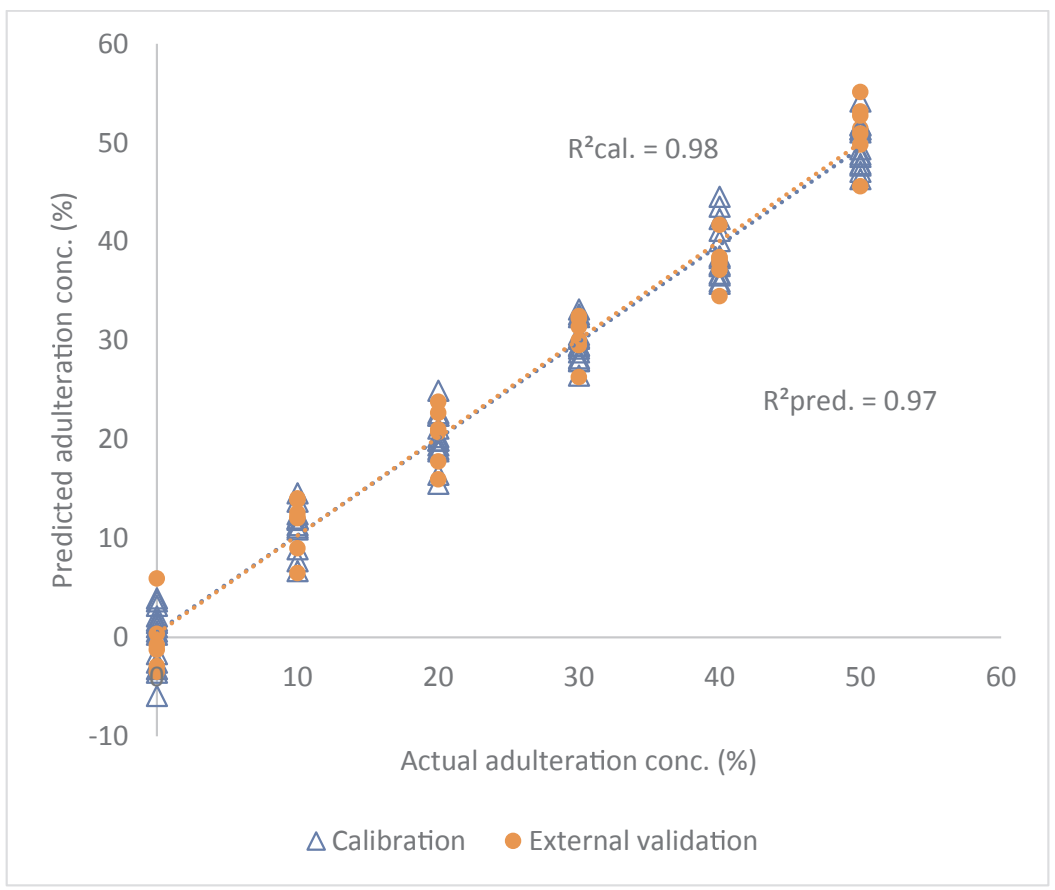

(b)

Fig. 3. Actual versus predicted percentages of old olive oil adulteration ( $0 \%$ to $50 \%$ v/v) determined by (a) FT-IR + UV-vis and (b) fluorescence spectroscopy.

that ratio as also indicated in a different type of adulteration study (Li, Wang, Zhao, Ouyang, \& Wu, 2015).

\section{Conclusion}

In the present study, it was aimed to develop reliable analytical tools to detect and quantify adulteration made with mixing fresh olive oils with old olive oil samples. Different spectroscopic approaches individually and as a combination are compared with each other using multivariate statistical techniques. The results indicated that both fluorescence and combination of FT-IR and UV-vis spectral data are better than FT-IR and UV-vis spectroscopy alone in the determination of adulteration due to their lower error values for prediction $(2.82 \%$ and $5.20 \%$, respectively) as well as their higher regression coefficients of prediction (0.97 and 0.91, orderly). Both UV-vis and FT-IR are rapid methods; however, collecting and analyzing the data statistically would require a longer time. However, even in this condition, using combined spectroscopy would have advantages over wet chemical analysis methods due to its minimal waste generating, no sample preparation and easy to use nature. Differentiation of adulterated samples are due to the presence of oxidation products and change in the pigment concentration of the oils. These methods could be used as reliable, fast, non-destructive, and environmentally friendly tools in both detection and quantification of adulteration as well as screening of olive oil 
quality, simultaneously.

\section{Conflicts of interest}

Authors declare no conflict of interest.

\section{Acknowledgements}

This study was supported by the Scientific and Technological Research Council of Turkey (Project No: TUBITAK TOVAG-115O584). We would like to thank Chemical Engineering Department and Environmental Research Center of Izmir Institute of Technology for their help in fluorescence spectroscopy and GC analyses, respectively.

\section{References}

Ali, H., Saleem, M., Anser, M. R., Khan, S., Ullah, R., \& Bilal, M. (2018). Validation of fluorescence spectroscopy to detect adulteration of edible oil in extra virgin olive oil (EVOO) by applying chemometrics. Applied Spectroscopy, 72, 1371-1379. https://doi. org $/ 10.1177 / 0003702818768485$.

Aroca-Santos, R., Cancilla, J. C., Pérez-Pérez, A., Moral, A., \& Torrecilla, J. S. (2016). Quantifying binary and ternary mixtures of monovarietal extra virgin olive oils with UV-vis absorption and chemometrics. Sensors and Actuators B: Chemical, 234, 115-121. https://doi.org/10.1016/j.snb.2016.04.094.

Borràs, E., Ferré, J., Boqué, R., Mestres, M., Aceña, L., \& Busto, O. (2015). Data fusion methodologies for food and beverage authentication and quality assessment-A review. Analytica Chimica Acta, 891, 1-14. https://doi.org/10.1016/j.aca.2015.04.042.

Carranco, N., Farrés-Cebrián, M., Saurina, J., \& Núñez, O. (2018). Authentication and quantitation of fraud in extra virgin olive oils based on HPLC-UV fingerprinting and multivariate calibration. Foods, 7, 44. https://doi.org/10.3390/foods7040044.

Cen, H., \& He, Y. (2007). Theory and application of near infrared reflectance spectroscopy in determination of food quality. Trends in Food Science \& Technology, 18, 72-83. https://doi.org/10.1016/j.tifs.2006.09.003.

EU (2016). Commission Delegated Regulation (EU) 2016/2095 of 26 September 2016 amending Regulation (EEC) No 2568/91 on the characteristics of olive oil and oliveresidue oil and on the relevant methods of analysis. Off. J. Eur. Union L, 326, 1-6.

EU (2012). Commission Implementing Regulation (EU) No 29/2012 of 13 January 2012 on marketing standards for olive oil. Official Journal of the European Union, L, 12, $14-21$.

EEC (1991). Commission Regulation (EEC) No 2568/91 of 11 July 1991 on the characteristics of olive oil and olive-residue oil and on the relevant methods of analysis. Official Journal of the European Communities - Legislation, 248, 1-83.

Danezis, G. P., Tsagkaris, A. S., Camin, F., Brusic, V., \& Georgiou, C. A. (2016). Food authentication: Techniques, trends \& emerging approaches. TRAC Trends in Analytical Chemistry, 85, 123-132. https://doi.org/10.1016/j.trac.2016.02.026.

Dankowska, A., \& Kowalewski, W. (2019). Comparison of different classification methods for analyzing fluorescence spectra to characterize type and freshness of olive oils. European Food Research and Technology, 245, 745-752. https://doi.org/10.1007/ s00217-018-3196-z.

Dankowska, A., \& Małecka, M. (2009). Application of synchronous fluorescence spectroscopy for determination of extra virgin olive oil adulteration. European Journal of Lipid Science and Technology, 111, 1233-1239. https://doi.org/10.1002/ejlt. 200800295.

Dupuy, N., Le Dréau, Y., Ollivier, D., Artaud, J., Pinatel, C., \& Kister, J. (2005). Origin of French virgin olive oil registered designation of origins predicted by chemometric analysis of synchronous excitation - emission fluorescence spectra. Journal of Agricultural and Food Chemistry, 53, 9361-9368. https://doi.org/10.1021/ jf051716m.

Engel, J., Gerretzen, J., Szymańska, E., Jansen, J. J., Downey, G., Blanchet, L., et al. (2013). Breaking with trends in pre-processing? TRAC Trends in Analytical Chemistry, 50, 96-106. https://doi.org/10.1016/j.trac.2013.04.015.

Galindo-Prieto, B., Eriksson, L., \& Trygg, J. (2015). Variable influence on projection (VIP) for OPLS models and its applicability in multivariate time series analysis. Chemometrics and Intelligent Laboratory Systems, 146, 297-304. https://doi.org/10. 1016/j.chemolab.2015.05.001.

Gurdeniz, G., \& Ozen, B. (2009). Detection of adulteration of extra-virgin olive oil by chemometric analysis of mid-infrared spectral data. Food Chemistry, 116, 519-525. https://doi.org/10.1016/j.foodchem.2009.02.068.

Hernández-Sánchez, N., Lleó, L., Ammari, F., Cuadrado, T. R., \& Roger, J. M. (2017). Fast fluorescence spectroscopy methodology to monitor the evolution of extra virgin olive oils under illumination. Food and Bioprocess Technology, 10, 949-961. https://doi. org/10.1007/s11947-017-1866-7.

Hirri, A., Bassbasi, M., Platikanov, S., Tauler, R., \& Oussama, A. (2016). FTIR spectroscopy and PLS-DA classification and prediction of four commercial grade virgin olive oils from Morocco. Food Analytical Methods, 9, 974-981. https://doi.org/10.1007/ s12161-015-0255-y.

Hirri, A., Gammouh, M., Gorfti, A., Kzaiber, F., Bassbasi, M., Souhassou, S., et al. (2015). The use of Fourier transform mid infrared (FT-MIR) spectroscopy for detection and estimation of extra virgin olive oil adulteration with old olive oil. Sky Journal of Food Science, 4, 60-66.

Jabeur, H., Drira, M., Rebai, A., \& Bouaziz, M. (2017). Putative markers of adulteration of higher-grade olive oil with less expensive pomace olive oil identified by gas chromatography combined with chemometrics. Journal of Agricultural and Food Chemistry, 65, 5375-5383. https://doi.org/10.1021/acs.jafc.7b00687.

Jabeur, H., Zribi, A., \& Bouaziz, M. (2016). Extra-virgin olive oil and cheap vegetable oils: Distinction and detection of adulteration as determined by GC and chemometrics. Food Analytical Methods, 9, 712-723. https://doi.org/10.1007/s12161-015-0249-9.

Jabeur, H., Zribi, A., Makni, J., Rebai, A., Abdelhedi, R., \& Bouaziz, M. (2014). Detection of Chemlali extra-virgin olive oil adulteration mixed with soybean oil, corn oil, and sunflower oil by using GC and HPLC. Journal of Agricultural and Food Chemistry, 62, 4893-4904. https://doi.org/10.1021/jf500571n.

Jiang, L., Zheng, H., \& Lu, H. (2015). Application of UV spectrometry and chemometric models for detecting olive oil-vegetable oil blends adulteration. Journal of Food Science \& Technology, 52, 479-485. https://doi.org/10.1007/s13197-013-1003-1.

Jiménez-Carvelo, A. M., Lozano, V. A., \& Olivieri, A. C. (2019). Comparative chemometric analysis of fluorescence and near infrared spectroscopies for authenticity confirmation and geographical origin of Argentinean extra virgin olive oils. Food Control, 96, 22-28. https://doi.org/10.1016/j.foodcont.2018.08.024.

Jiménez-Carvelo, A. M., Osorio, M. T., Koidis, A., González-Casado, A., \& CuadrosRodríguez, L. (2017). Chemometric classification and quantification of olive oil in blends with any edible vegetable oils using FTIR-ATR and Raman spectroscopy. $L W T$ Food Science Technology, 86, 174-184. https://doi.org/10.1016/j.lwt.2017.07.050.

Jolayemi, O. S., Tokatli, F., Buratti, S., \& Alamprese, C. (2017). Discriminative capacities of infrared spectroscopy and e-nose on Turkish olive oils. European Food Research and Technology, 243, 2035-2042. https://doi.org/10.1007/s00217-017-2909-z.

Lastra-Mejías, M., Aroca-Santos, R., Izquierdo, M., Cancilla, J. C., Mena, M. L., \& Torrecilla, J. S. (2019). Chaotic parameters from fluorescence spectra to resolve fraudulent mixtures of fresh and expired protected designation of origin extra virgin olive oils. Talanta, 195, 1-7. https://doi.org/10.1016/j.talanta.2018.10.102.

Li, X., \& Wang, S. C. (2018). Shelf life of extra virgin olive oil and its prediction models. Journal of Food Quality, 2018. https://doi.org/10.1155/2018/1639260.

Li, B., Wang, H., Zhao, Q., Ouyang, J., \& Wu, Y. (2015). Rapid detection of authenticity and adulteration of walnut oil by FTIR and fluorescence spectroscopy: A comparative study. Food Chemistry, 181, 25-30. https://doi.org/10.1016/j.foodchem.2015.02. 079.

Li, Y., Xiong, Y., \& Min, S. (2019). Data fusion strategy in quantitative analysis of spectroscopy relevant to olive oil adulteration. Vibrational Spectroscopy, 101, 20-27. https://doi.org/10.1016/j.vibspec.2018.12.009.

Lleó, L., Hernández-Sánchez, N., Ammari, F., \& Roger, J. M. (2016). 3D front-face fluorescence spectroscopy for characterization of extra virgin olive oil and olive oil according to the spectral pattern. Agricultural Engineering International: CIGR Journal, 18, 190-199.

Merás, I. D., Manzano, J. D., Rodríguez, D. A., \& de la Peña, A. M. (2018). Detection and quantification of extra virgin olive oil adulteration by means of autofluorescence excitation-emission profiles combined with multi-way classification. Talanta, 178, 751-762. https://doi.org/10.1016/j.talanta.2017.09.095.

Mignani, A. G., Ciaccheri, L., Mencaglia, A. A., \& Cimato, A. (2012). Optical absorption spectroscopy for quality assessment of extra virgin olive oil. In D. Boskou (Ed.). Olive oil-constituents, quality, health properties and bioconversions (pp. 47-62). Rijeka, Croatia: Intech.

Moros, J., Garrigues, S., \& de la Guardia, M. (2010). Vibrational spectroscopy provides a green tool for multi-component analysis. TRAC Trends in Analytical Chemistry, 29, 578-591. https://doi.org/10.1016/j.trac.2009.12.012.

Öztürk, B., Ankan, A., \& Özdemir, D. (2010). Olive oil adulteration with sunflower and corn oil using molecular fluorescence spectroscopy. In V. R. Preedy, \& R. R. Watson (Eds.). Olives and olive oil in health and disease prevention (pp. 451-461). Oxford, UK: Academic press.

Ozturk, B., Yucesoy, D., \& Ozen, B. (2012). Application of mid-infrared spectroscopy for the measurement of several quality parameters of alcoholic beverages, wine and raki. Food Analytical Methods, 5, 1435-1442. https://doi.org/10.1007/s12161-0129397-3.

Riedl, J., Esslinger, S., \& Fauhl-Hassek, C. (2015). Review of validation and reporting of non-targeted fingerprinting approaches for food authentication. Analytica Chimica Acta, 885, 17-32. https://doi.org/10.1016/j.aca.2015.06.003.

Rohman, A., Che Man, Y. B., Ismail, A., \& Hashim, P. (2017). FTIR spectroscopy coupled with chemometrics of multivariate calibration and discriminant analysis for authentication of extra virgin olive oil. International Journal of Food Properties, 20 S1173-S1181. https://doi.org/10.1080/10942912.2017.1336718.

Sen, I., \& Tokatli, F. (2016). Differentiation of wines with the use of combined data of UV-visible spectra and color characteristics. Journal of Food Composition and Analysis, 45, 101-107. https://doi.org/10.1016/j.jfca.2015.09.018.

Sinelli, N., Cosio, M. S., Gigliotti, C., \& Casiraghi, E. (2007). Preliminary study on application of mid infrared spectroscopy for the evaluation of the virgin olive oil "freshness". Analytica Chimica Acta, 598, 128-134. https://doi.org/10.1016/j.aca. 2007.07.024.

Sun, X., Lin, W., Li, X., Shen, Q., \& Luo, H. (2015). Detection and quantification of extra virgin olive oil adulteration with edible oils by FT-IR spectroscopy and chemometrics. Analytical Methods, 7, 3939-3945. https://doi.org/10.1039/C5AY00472A.

Tamaki, Y., \& Mazza, G. (2011). Rapid determination of lignin content of straw using Fourier Transform Mid-Infrared Spectroscopy. Journal of Agricultural and Food Chemistry, 59, 504-512. https://doi.org/10.1021/jf1036678.

Tan, J., Li, R., Jiang, Z., Shi, M., Xiao, Y., Jia, B., et al. (2018). Detection of extra virgin olive oil adulteration with edible oils using front-face fluorescence and visible spectroscopies. Journal of the American Oil Chemists Society, 95, 535-546. https://doi. org/10.1002/aocs.12071.

Tena, N., Aparicio, R., \& García-González, D. L. (2018). Photooxidation effect in liquid lipid matrices: Answers from an innovative FTIR spectroscopy strategy with "mesh 
cell" incubation. Journal of Agricultural and Food Chemistry, 66, 3541-3549. https:// doi.org/10.1021/acs.jafc.7b05981.

Torreblanca-Zanca, A., Aroca-Santos, R., Lastra-Mejías, M., Izquierdo, M., Cancilla, J. C., \& Torrecilla, J. S. (2019). Laser diode induced excitation of PDO extra virgin olive oils for cognitive authentication and fraud detection. Sensors and Actuators B: Chemical, 280, 1-9. https://doi.org/10.1016/j.snb.2018.10.014.

Torrecilla, J. S., Rojo, E., Domínguez, J. C., \& Rodríguez, F. (2010). A novel method to quantify the adulteration of extra virgin olive oil with low-grade olive oils by UV vis. Journal of Agricultural and Food Chemistry, 58, 1679-1684. https://doi.org/10. 1021/jf903308u.

Uncu, O., \& Ozen, B. (2015). Prediction of various chemical parameters of olive oils with Fourier transform infrared spectroscopy. LWT-Food Science and Technology, 63, 978-984. https://doi.org/10.1016/j.lwt.2015.05.002.

Valli, E., Bendini, A., Berardinelli, A., Ragni, L., Riccò, B., Grossi, M., et al. (2016). Rapid and innovative instrumental approaches for quality and authenticity of olive oils. European Journal of Lipid Science and Technology, 118, 1601-1619. https://doi.org/ 10.1002/ejlt.201600065. 